\title{
Thyroxine treatment: absorption, malabsorption, and novel therapeutic approaches
}

\author{
Marco Centanni
}

Received: 26 September 2012/ Accepted: 3 October 2012/Published online: 25 October 2012

(C) Springer Science+Business Media New York 2012

\begin{abstract}
Although levothyroxine sodium is highly prescribed worldwide, the exact daily dosage, mode of assumption, and refractoriness to treatment are as yet matter of debate. Growing evidence highlighted that undertreatment with T4 has detrimental effects on several tissues and functions. This is even more proper during pregnancy, when an inadequate treatment may increase the likelihood of obstetric complications and may affect the neuropsychological performance during childhood [1]. In adult patients, subclinical hypothyroidism has been reported to be involved in impaired lipid metabolism and atherosclerosis as well as in cardiovascular disorders [2]. The need for an individually tailored dose potently emerges from these findings. Thyroxine treatment should no longer be prescribed according to the commercially available dose size, but rather related to the patient's weight and age [3]. Two major issues are indeed crucial to obtain an optimal daily dose for a specific patient: an unbiased serum TSH value and an efficient absorption process of thyroxine. The assumption that serum TSH would be a reliable marker of pharmacologic euthyroidism in all tissues has been challenged since its value may be affected by a number of drugs, cosmetics, and pathophysiological conditions [4, 5]. Even the upper limit of normal serum TSH $(<$ or $>2.5 \mathrm{mU} / \mathrm{l})$ has been questioned [5]. However, it still remains the best available marker, since the direct
\end{abstract}

M. Centanni

Chief of Endocrinology Unit, Department of Medico-Surgical Sciences and Biotechnologies, Sapienza University of Rome, Latina, Italy

M. Centanni ( $\square)$

Dipartimento di Medicina Sperimentale, Policlinico Umberto I, Viale Regina Elena 324, 00161 Rome, Italy

e-mail: marco.centanni@uniroma1.it measurement of thyroxine absorption is not an easy task. Assuming an unbiased serum TSH value in each patient, the absorption process of $\mathrm{T} 4$ becomes key for a successful treatment. The daily dose required to obtain the desired $\mathrm{TSH}$ value/therapeutic effect is, in fact, not simply a mirror of the ingested dose of thyroxine. One of the major determinants on pharmacological thyroid homeostasis is, thus the incomplete absorption of oral thyroxine (60-80\% of the administered dose) which takes place at the intestinal level. Indeed, the absorbed dose of T4 may be considered the resultant of: (a) patient compliance; (b) some physiological (pregnancy, weight) and/or paraphysiological (behavioral, nutritional) or pharmacological conditions of increased or decreased T4 need; and (c) the presence of diseases which cause malabsorption of $\mathrm{T} 4$ (see [6] for review). Repeated failure to reach therapeutic target in response to an "adjusted" dose is a fairly common and frustrating experience. Larger doses of thyroxine are required to attain the desired serum TSH concentrations, leading to expensive and often inappropriate hormonal monitoring. Once attributed to pseudomalabsorption, the increased need for thyroxine may be also explained by an impaired absorption of levothyroxine. Recently, the modality of drug ingestion gained attention. There is now evidence that at least $1 \mathrm{~h}$ delay between $\mathrm{T} 4$ tablet ingestion and breakfast warrant the best therapeutic achievement [7]. According to Benvenga et al. [8], even the common use of coffee close to the T4 tablet ingestion results in an altered T4 absorption. According to these authors, coffee physically interacts with $\mathrm{T} 4$ tablets and retains thyroxine within the intestinal lumen thus making it less available for absorption [8]. This mechanism closely resembles the one suggested for the reduced absorption of thyroxine in patients with celiac sprue [9]. In these patients, the amount of active hormone available for the absorption may be 
reduced by partially undigested substances, sequestering thyroxine in the intestinal lumen [9]. In this issue of the Journal, Vita et al. [10] seem to have overcome the problem of coffee interference using a soft gel capsules preparation containing T4 dissolved in glycerin. They presented evidence that, unlike tablet formulation of $\mathrm{T} 4$, absorption of soft gel capsules is unaffected by the concurrent assumption of coffee. If the soft gel capsules are able to bypass the sequestering mechanism of $\mathrm{T} 4$ within the intestinal lumen, then they may be of potential benefit also in celiac patients. Absorption of T4 may be similarly improved in patients with lactose intolerance and other chronic inflammatory, disorders in which intestinal malabsorption of T4 may also occur.

Whatever would be the involved mechanism, these findings and the growing evidence of thyroxine malabsorption may lead to reconsider the whole picture of thyroxine treatments. Further important characteristic of this novel formulation is an improved $\mathrm{pH}$-dependent dissolution profile, as compared to tablets [11]. The increased need for thyroxine in patients with impaired gastric acid secretion has been described since 2006 [12] and a novel role for the stomach in the subsequent intestinal $\mathrm{T} 4$ tablet absorption was also highlighted. If a preparation of oral $\mathrm{T} 4$ better dissolves in less acidic environment, then the absorption process may be improved in those patients with impaired gastric acid secretion. Indeed, the gastric acid producing machinery is partially or totally damaged in patients with gastric atrophy and/or with Helicobacter pylori infection (in which is also challenged by $\mathrm{NH} 3$ production) and is blocked in those treated with proton pump inhibitors (PPI). The huge number of patients with impaired acid secretion and potential $\mathrm{T} 4$ malabsorption (30\% of patients in Western Countries have H. pylori infection and PPI are among the most prescribed drugs) may benefit from this novel thyroxine preparation. On this ground, a further step, beside the tablets and the soft gel capsules, may be the therapeutic option represented by the oral liquid preparation of T4.

In vivo studies confirming that the use of more soluble T4 preparations correspond to a more effective treatment in patients with gastrointestinal disorders are still awaited.
Indeed, the findings of Vita and coll., published in this issue, indicate the need for different $\mathrm{T} 4$ preparations available to meet the habits and the therapeutic needs of different class of patients.

\section{References}

1. L. Kooistra, S. Crawford, A.L. van Baar, E.P. Brouwers, V.J. Pop, Neonatal effects of maternal hypothyroxinemia during early pregnancy. Pediatrics 117, 161-167 (2006)

2. B. Biondi, I. Klein, Hypothyroidism as a risk factor for cardiovascular disease. Endocrine 24, 1-13 (2004)

3. J. Jonklaas, Sex and age differences in levothyroxine dosage requirement. Endocr. Pract. 16, 71-79 (2010)

4. B.R. Haugen, Drugs that suppress TSH or cause central hypothyroidism. Best. Pract. Res. Clin. Endocrinol. Metab. 23, 793-800 (2009)

5. L. Wartofsky, R.A. Dickey, The evidence for a narrower thyrotropin reference range is compelling. J. Clin. Endocrinol. Metab. 90, 5483-5488 (2005)

6. M. Centanni, M.G. Santaguida, L. Gargano: Malabsorption of T4: new insights on oral thyroxine treatment. Hot Thyroidol. 169. http://www.hotthyroidology.com. Accessed 1-4 June 2007

7. T.G. Bach-Huynh, B. Nayak, J. Loh, S. Soldin, J. Jonklaas, Timing of levothyroxine administration affects serum thyrotropin concentration. J. Clin. Endocrinol. Metab. 94, 3905-3912 (2009)

8. S. Benvenga, L. Bartolone, M.A. Pappalardo, A. Russo, D. Lapa, G. Giorgianni, G. Saraceno, F. Trimarchi, Altered intestinal absorption of L-thyroxine caused by coffee. Thyroid. 18, 293-301 (2008)

9. C. Virili, G. Bassotti, M.G. Santaguida, R. Iuorio, S.C. Del Duca, V. Mercuri, A. Picarelli, P. Gargiulo, L. Gargano, M. Centanni, Atypical celiac disease as cause of increased need for thyroxine: a systematic study. J. Clin. Endocrinol. Metab. 97, E419-E422 (2012)

10. R. Vita, G. Saraceno, F. Trimarchi, S. Benvenga, A novel formulation of L-thyroxine (L-T4) reduces the problem of L-T4 malabsorption by coffee observed with traditional tablet formulations. Endocrine (2012). doi:10.1007/s12020-012-9772-2

11. D. Pabla, F. Akhlaghi, H.A. Zia, Comparative pH-dissolution profile study of selected commercial levothyroxine products using inductively coupled plasma mass spectrometry. Eur. J. Pharm. Biopharm. 72, 105-110 (2009)

12. M. Centanni, L. Gargano, G. Canettieri, N. Viceconti, A. Franchi, G. Delle Fave, B. Annibale, Thyroxine in goiter, Helicobacter pylori infection, and chronic gastritis. N. Engl. J. Med. 354, 1787-1795 (2006) 